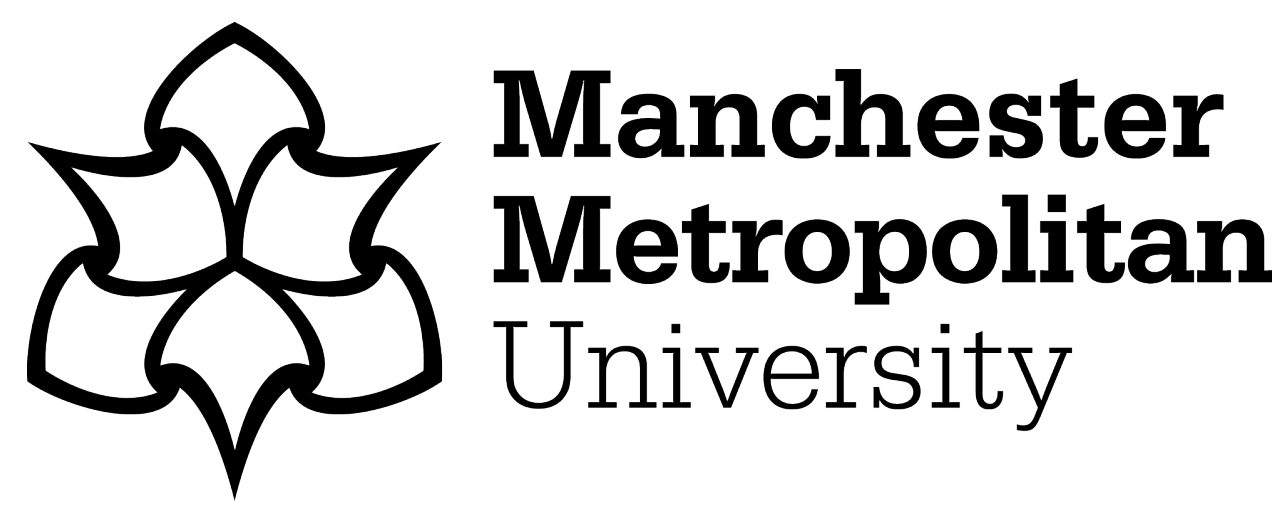

Wigham, P and Challis, B ORCID logoORCID: https://orcid.org/0000-00020200-8087 (2020) Real-time measurement and analysis of audience response. In: ArtsIT 2019 - 8th EAI International Conference: ArtsIT, Interactivity \& Game Creation, 06 November 2019 - 08 November 2019, Aalborg, Denmark.

Downloaded from: https://e-space.mmu.ac.uk/627204/

Publisher: Springer

DOI: https://doi.org/10.1007/978-3-030-53294-9_4

Please cite the published version 


\title{
Real-time Measurement and Analysis of Audience Response
}

\author{
Philip Wigham and Ben Challis \\ Manchester Metropolitan University, Manchester, UK \\ \{p.wigham, b. challis\}@mmu . ac.uk
}

\begin{abstract}
How do you harness a "level" of emotional connectivity from audience/participants? Questionnaires, focus group discussions, interviews and other qualitative methods gather retrospective thoughts of the participant and may miss important insights or connections that could be discovered if a real-time response is recorded. The aspiration for real-time audience data recording is problematic in many areas of research, in particular performing arts where the work/research presented is time bound. In addressing this problem within research into the design of novel musical controllers, custom "sliders" were used to measure and examine real-time audience response to short musical performances. The audience moved their sliders in response to the performance, producing continuous data that was recorded into music software and timestamped. The initial test results have shown promising insights and usefulness for real-time data collection and examination. These results and possible methods of data analysis are presented along with discussion on how this approach may be applied in other research contexts.
\end{abstract}

Keywords: Audience $\cdot$ Response $\cdot$ Real-time $\cdot$ Measurement $\cdot$ Mimesis - Mimetic theory · Performance · Music · Haptic · Sensory · Kinaesthetic - Tangibility · Feedback · Analysis · Data

\section{Introduction}

This paper presents a possible solution to the problematic area of recording continuous real-time audience response data. It is hoped that it will prove to be a useful data gathering tool in areas of research looking to acquire this type of data. The conception, implementation and initial results of a real-time "slider" mechanism for examining audience/participant response, will be discussed further along with its application in the current research, and how the slider method may be applied in other contexts.

The test phases being described in this paper were conceived as a way of recording and examining real-time audience response to new mimetic digital music instruments (DMI) and comparing them with existing traditional DMIs. Custom built "sliders" were used to allow audience/participants to respond to musical performances. The slider is moved up or down in response to a question posed before the performance, in this case how "engaged/interested" they are 
at each moment of the performance. As the participant feels more engaged they respond by moving the slider upwards, and downwards when less engaged. This "slider" data was captured in real-time and then collated with other data collected through the questionnaires and group discussions. The slider data proved crucial to gaining further insight into the qualitative questionnaires and focus group discussions.

This method is proving to be an effective way to examine a participant's continually adjusting response to a live performance. The slider data has highlighted participant responses to the performance that did not appear within the questionnaires or post-performance discussions. This allowed further examination of those areas of the performance and instigated changes to the performance. Further testing showed that these changes in the performance had affected the slider data and therefore the audience response.

Although the mimetic design study is in its very early stages, the results are already showing very interesting and useful insights, which will be discussed in more detail below.

\section{Research Context}

The need for real-time participant response data came about through post graduate research into mimetic influenced digital music instrument design. The research has been comparing user perceptions of music performances using a custom-built MIDI ${ }^{1}$ controller (inspired by mimetic theories[21]) alongside a traditional MIDI keyboard controller. This has required a method of data collection and examination of participant response to music performances. Each MIDI controller was connected to the same laptop running a software synthesiser which both controllers were able to play and control. A musical performance was devised that could be played with both controllers so that the sound elements of each performance were as identical as possible. This allowed for a fairer comparison of the actual physical and gestural performance of the instruments.

Cox's mimetic hypothesis[12] identifies overt and covert 'mimetic participation' as audience responses to musical performance. It was desirable to find a way to explore how audience participants may experience mimetic participation and though overt manifestations could be observed in the video, covert mimetic participation, by its nature, does not manifest itself. Though questionnaires and interviews were initially devised, it was important to find an effective method of investigating real-time response to discover any covert "mimetic participation" [11,12]. Any post-performance data collection such as questionnaires and focus group discussions, being retrospective, may miss exposing this covert mimetic participation and any allusion to mimetic participation could not be precisely associated with an exact moment in the performance.

\footnotetext{
1 'MIDI is an industry standard music technology protocol that connects products from many different companies including digital musical instruments, computers, tablets, and smartphones.'[1]
} 
In addressing this problem, a "slider" method was developed to enable response data to be recorded in real-time during the performances, which allowed audience members to respond continuously to how "engaged" they felt throughout the performance. The data from these sliders was recorded into a MIDI recording software ${ }^{2}$ as MIDI data, simultaneously with the performance MIDI data, whilst two video cameras captured the audience and performer. This allowed the slider data to then be compared with various points within the performance, achieving continuous real-time snapshots of the level of audience engagement at each moment of the performance. This data was also compared with the demographic data, the post-performance questionnaires and the focus group discussions.

Although the findings from the mimetic design research are in the early stages of analysis, this quantitative slider data is providing more insight than any other isolated method, and is helping to expose moments of potential covert mimetic participation, that would have otherwise remain hidden. Synchronising the slider data to the video recordings allows for a very direct analysis of the performance in relation to the audience response. This slider method of data collection and analysis is discussed in more detail below.

\section{Methodology}

\subsection{Existing Methods}

The problem of recording real-time audience response data has been approached using several methods[10], the most prevalent of which include analysis of audience biometric data, detection of audience motion, and input devices for audience response.

The advantage of recording biometric data is its involuntary nature requiring no conscious effort to respond, therefore being completely unobtrusive to the experience of the performance. Galvanic Skin Response (GSR) and electrodermal activity have been used to suggest a biological response to performances[20,14], as well as cardiovascular and respiratory measurements[9]. However it remains difficult to convincingly associate biometric data directly with a specific response to the performance, such as engagement. Latulipe et al.[13] attempted to resolve this issue by comparing audience GSR data with 'self-report scales' finding a 'strong correlation'. However, there are still problems with interpreting biometric data with any certainty in relation to audience levels of engagement.

Martella et al.[16] used accelerometers and infra-red sensors to record audience movement during a live dance performance to predict the outcome of post-performance questionnaires and motion capture techniques were used by Swarbrick et al. [19] to investigate audience response to live performances compared with recorded music. As with the biometric data techniques there is a difficulty with being able to directly link the movement of audience members

\footnotetext{
${ }^{2}$ For the purposes of this study, Ableton Live was used, though it would be feasible to use other digital audio workstations or MIDI recording software.
} 
with a response to the performance, and although these methods have the similar advantage of being unobtrusive, they are difficult to interpret precisely.

The portable Audience Response Facility (pARF) utilises a personal digital assistant (PDA) as an input device[17] which allows input of two simultaneous data streams using the PDA stylus on an $\mathrm{X}$ and $\mathrm{Y}$ axis. This allows the audience to be asked to respond in a particular way, providing participant responses that are explicitly connected with the posed response parameter, such as "engagement". However, the stylus/PDA input device could be potentially distracting to the participant, having to look at the PDA to give an accurate response.

Stevens et al.[18] suggest that other suitable input devices might be used depending on the response being measured. Other input devices could include commercially available keypads $[6,4]$ and mobile phone apps $[7,2]$. The keypads capture real-time data but only have the capability of an on/off style button response, and therefore do not give a scaled response. Although there are a range of mobile apps that can be used to collect audience response data, most of them cannot track scaled response. Reactor[5] is a mobile app that can record a scaled response via a slider-bar on the screen, but is aimed at pre-recorded video not live performance. Critically, mobile phone input could be a potential distraction to the user, having to glance down at the phone regularly to gauge their position on the screen.

The mimetic design project required a bespoke solution due to the specific context of the research. The music performance, not intrinsically an "event", was created to exploit the gestural nature of the DMIs. It was necessary to be able to scrutinise the detail behind individual participants, not a consensus across the audience, exploring the how, when and why of apparent response by individuals to specific gestures. In practice, during the testing phase using bespoke physical sliders, it was common for the participants to turn their mobile devices off (not having been previously encouraged to do so) and hold the slider in one hand, being entirely prepared for the task. The tactile and tangible nature of the physical slider meant that the participants could fully concentrate on the performance whilst also confidently controlling the slider.

\section{$3.2 \quad$ Sliders}

The sliders were made using a linear slide potentiometer soldered to an XLR socket. This was housed in a wooden enclosure designed to fit the size of an average hand, so that the thumb could move the slider (see Fig. 1). Ten sliders were made and connected with a cable to a micro controller unit (MCU). The MCU converts the movement of the potentiometers into digital signals through an ADC and then outputs this data in the MIDI format via USB interface. The MIDI protocol was chosen due to the availability of well established MIDI recording software capable of recording multiple streams of slider data.

It proved to be important to record calibration data (see Fig. 2 below) of the participants slider movement before any actual performance recordings took place. This involved asking the participants to move the slider as far to the top as they comfortably could and to the bottom again as far as they could, 


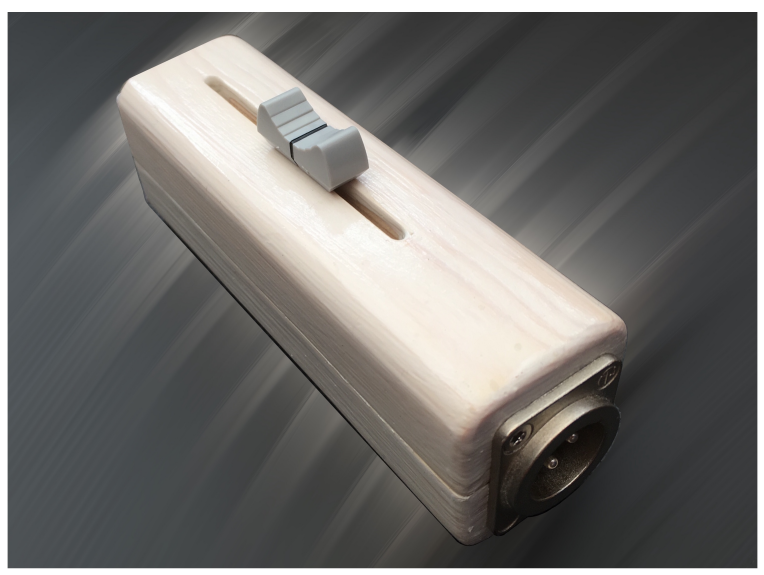

Fig. 1. Custom Slider

and lastly to the middle. This made an important allowance for people who may have a limited slider range due to smaller hands or restricted movement. This calibration procedure was completed before each recording, due to the possibility that participants may start from a different "middle" position each time. Without this calibration the accuracy between multiple recordings and the recordings of other participants would be compromised.

Implementation of the sliders requires preparing the participants with the parameters for response, and the question they are to respond to with the slider. In this research the participants were asked to respond to how "engaged" they felt during the musical performance and to continually respond as necessary, moving the slider upwards as they felt more engaged and down when they were less engaged. The slider could, of course, be used with any question, providing illumination into many possible areas of audience response. It may be presented in a similar context to the standard Likert[15] scale, the middle being neither agree/disagree with the posed question, higher slider positions equating to more agreement and lower levels to disagreement. This data can then be analysed in the same way as Likert response data from questionnaires, the difference being that it is real-time and continuously changing with the response of the participant.

\section{Data Analysis}

\subsection{Slider Data}

Although the MCU could be configured to output the data in different formats, such as ascii sent via a serial connection, MIDI data is a convenient and well established way of recording the data in real-time. Once recorded, the MIDI slider data can be analysed in several ways. The slider data is recorded as MIDI 
Continuous Controller (CC) data which has a range of 0 to 127 (7 bit). There are 128 independent CC's available, and each slider is recorded to an individual CC. Most MIDI recording software allows the MIDI data to be exported as a standard MIDI file (SMF). ${ }^{3}$ These files add a timestamp to the recorded MIDI data to allow the data to be played back accurately.

The SMF file can be converted to text/csv and imported into a standard database and/or spreadsheet for further analysis. Fig. 2, below, shows two participant's slider data streams, overlaid on the same graph to allow comparison of levels of engagement over the period of the performance. The calibration process can be clearly seen on this graph with the initial high and low levels. The data for

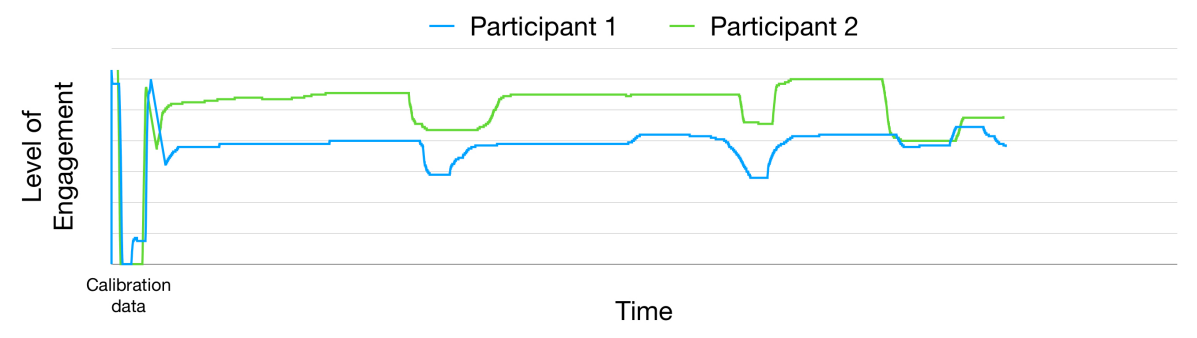

Fig. 2. Slider Data of Two Participants

each participant can be separated out, (due to the individually assigned CC's) and analysed individually. This is useful if slider data between two or more performances is to be analysed. This allows the data from each performance from the same participant to be examined.

The MIDI recording of a musical performance may be recorded directly from the MIDI controller alongside the slider data. This data can be processed in the same way as the slider data and then used to compare audience response with the musical gestures of the performance. This may also be possible wherever sensors/devices capable of producing MIDI data are used in the performance. This is a useful method of analysis for performances using such technology, however, synchronising the slider data to the video recording is a more universal approach, allowing the data to be analysed directly with the recorded performance. This approach will now be discussed in more detail.

\subsection{Video Synchronisation}

The MIDI slider data can be recorded into any MIDI capable software. To allow the slider data to be synchronised to the video of the performance, a sound/click

\footnotetext{
3 'Standard MIDI Files contain all the MIDI instructions to generate notes, control individuals volumes, select instrument sounds, and even control reverb and other effects.'[1]
} 
should be placed at the beginning of the recording session in the recording software. This sound will then play at the start of the MIDI recording and will be heard on the video recording allowing synchronisation of the data with the video. A simpler alternative would be to make a "sync" sound or speak a specific word at the same time as starting the MIDI recording, which would serve the same purpose, as an anchor point to sync the MIDI recording with the video. MIDI software commonly synchronises with video editing software using the SMPTE (Society of Motion Picture and Television Engineers)[8] protocol. This would allow the MIDI software to playback the slider data concurrently with software running the video. This requires two separate software applications: one for MIDI and one for the video. An alternative method, requiring only one application, would be to use MIDI software capable of importing video, allowing the initial "sync" sound, to be aligned with the beginning of the recording. The slider data can then be played back along with the video, within the same software package.

\subsection{Video with Slider Data Overlay}

The methods of analysis described above both require specific software to view the data. A more accessible and potentially more useful way of processing the slider data with video, is to create a video that overlays the continuous slider data on top of the video recording of the performance. This video can then be viewed in any application capable of video playback, allowing more convenient analysis and sharing of data. This provides a very visual way of viewing the data in direct relation to the performance.

Firstly a video recording of the slider data playback needs creating. If the MIDI recording software has its focus set to follow the playback cursor, a video screen capture of the slider data playing back can be made. Using video editing software, this video can then be overlaid and aligned with the start/click sound on the performance video, allowing the movement of the slider data to be observed with the exact moment in the performance video when it occurred. Fig. 3 shows the red play cursor of the slider data and how that data has moved along with the performance video. This provides a very powerful real-time analysis
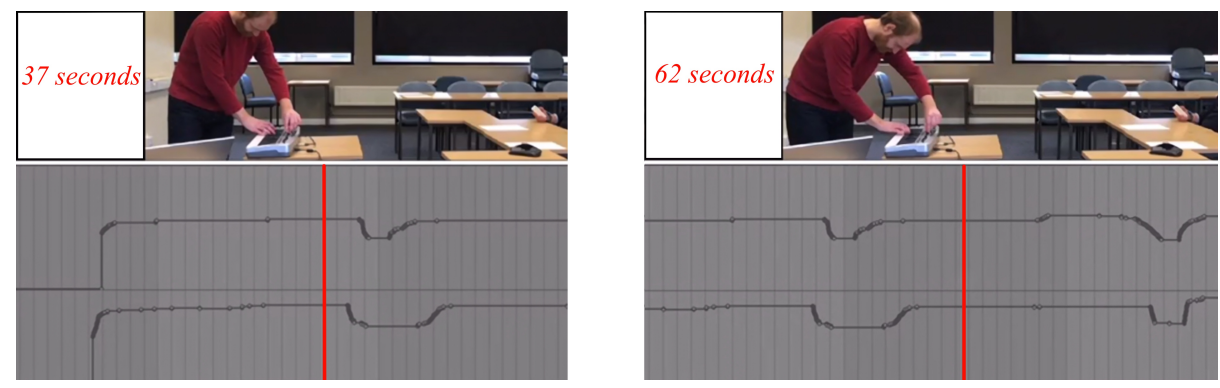

Fig. 3. Video with Slider Overlay 
of the posed slider question, whether that is level of engagement, excitement, interest, immersion within a space or an evaluation of film, TV, gameplay, performance, dance, radio, theatre, etc.

\section{Discussion}

Although the mimetic design research is in the early stages, this "slider" method is already proving to be a powerful way of acquiring and analysing real-time data. A good example of this is provided in Fig. 2 which shows three distinctive dips for both participants, highlighting an area of interest requiring further examination. When comparing these points with the video data, it showed the dips occurring every time there was a slight pause as the synthesiser sound was changed. Neither of the participants eluded to this in the post-performance questionnaires. This discovery was only possible by finding out exactly where in the performance these dips occurred. As a consequence the performance was adjusted so there were no gaps, creating a more fluid performance from one sound to another. Slider data from this adjusted performance showed no more dips occurring on the sound change overs.

It is possible that even with showing a participant the slider data after the performance they wouldn't remember what they were responding to at that point, especially with longer performances. This demonstrates the usefulness of being able to analyse the slider data in real-time. Fig. 3 shows the first minute of the same participant slider results, overlaid onto the performance video. This photograph demonstrates how easily the slider response data from several participants can be compared with each other and the exact point of occurrence in the performance video. This is particularly useful when the performances have an important visual aspect, in this case the gestural movements of playing instruments.

Since, this initial test with two participants, the slider method has been tested with more participants and larger groups. This data has only recently been taken, and so has not been fully analysed, but is showing promising signs of similar discoveries. There were some improvements made from the initial test including the addition of questionnaires specific to the research, labelled with the number of the corresponding participant slider. The hope is to provide deeper insight into the slider data, looking at how participant demographics and musical experience may affect the way they respond to music performance. Post-performance focus group discussions are being digitally transcribed, enabling the open responses to be considered with the slider data.

\section{Conclusion and Future Directions}

\subsection{Evaluation and Future Improvements}

The results so far indicate that the slider method for recording and examining continuous real-time response data is a viable technique. However, more research 
is necessary to fully investigate its potential and explore other possible uses. Since the initial testing phase, several points have come in to focus, including some future improvements.

The calibration before each recording is crucial to successful comparison of results. Fig. 2 shows that participant 2 has a higher initial "middle" slider position than participant 1, which needs to be taken into account when comparing levels. Adjustments to overall levels can be made using this calibration data, and is necessary for each recording because the "middle" point for a participant can vary between performances.

The sliders work well for groups of up to ten, but with larger groups the cabling to each slider might become less manageable. This leads to the consideration of a wireless system, and of course points to a possible use of mobile phones as a replacement to the sliders, due to the ease of implementing a wireless configuration. However, as mentioned previously, the physical sliders provide a tactile, intuitive interface for audience response, that generates continuous input without unnecessary distraction from the performance. The tangibility of the sliders more than compensates for the complexity of developing a custom wireless slider system and with the availability of wireless mesh network technologies is not a huge issue to overcome. A wireless system would allow a larger audience participation, and with the MIDI system having 16 separate channels of input as well as the 128 continuous controllers (discussed above) this would give a potential possibility of 2048 participants slider data being recorded simultaneously.

MIDI data is a relatively old system and has a low resolution of 128 steps (7 bit). If a greater resolution is needed OSC[3] could be implemented, although it is not as well supported as MIDI. There is a new MIDI specification (MIDI 2.0) being developed which will provide much higher resolutions, but is still in a prototyping stage. It will be compatible with the original MIDI specification so it should be simple to accommodate the new MIDI 2.0 protocol into existing work, when it is finally confirmed and available.

Another consideration in terms of analysis of slider data would be to segment the data points into discrete groups i.e. dividing a scale of 0 to 100 into 5 groups: 0-20, 21-40, 41-60, 61-80, 81-100. These groups could then be analysed in a manner similar to a Likert scale using standard statistical methods.

\subsection{Other Use Contexts}

This paper does not present a completed project but is a contribution to facilitate other research, where the "slider" solution to recording real-time response data might prove to be a useful tool. It is advantageous for several reasons: the data recorded in real-time; the data being continuous, which may capture data that an on/off "button" response might otherwise miss; the tangibility of the slider; the potential for large participant groups; the possibilities for post-performance analysis and the integration and consolidation of this quantitative slider data with other available qualitative data. This method may also prove useful due to participants finding difficulty in describing a particular event they were responding to within the performance, during a post-performance questionnaire 
or interview. With the slider method the participant doesn't need to describe the moment because the response data is synchronised to that moment.

The slider method for recording real-time response data may be implemented in a variety of contexts where a continuous real-time response from participants is required. This may be useful for research in any situation requiring realtime response data, such as media, film, gameplay, etc., but in particular is well suited to live performances in theatre, dance, music etc. A dance performance, for example, may also record data from sensors attached to the dancer, allowing the gestures within the dance to be analysed with the slider data.

It is clear from these initial explorations that the slider method is worth further investigation and development, and it is hoped that it may provide an efficacious tool for other research.

\section{References}

1. MIDI Association (2017), https://www.midi.org/

2. Mentimeter (2019), https://www.mentimeter.com/

3. Open Sound Control (2019), http://www.opensoundcontrol.org

4. Optivote (2019), https://www.optivote.co.uk/

5. Reactor (2019), http://www.roymorgan.com/products/reactor

6. Reply systems (2019), https://www.replysystems.com/

7. sli.do (2019), https://www.sli.do/

8. We are SMPTE | Society of Motion Picture \& Television Engineers (2019), https://www.smpte.org/

9. Bachrach, A., Fontbonne, Y., Joufflineau, C., Ulloa, J.L.: Audience entrainment during live contemporary dance performance: physiological and cognitive measures. Front. Hum. Neurosci. 9(179) (may 2015). https://doi.org/10.3389/fnhum.2015.00179

10. Brown, S., Hutton, A.: Developments in the real-time evaluation of audience behaviour at planned events. International Journal of Event and Festival Management 4, 43-55 (may 2013). https://doi.org/10.1108/17582951311307502

11. Cox, A.: Hearing, Feeling, Grasping Gestures. In: Gritten, A., King, E. (eds.) Music and Gesture, pp. 45-60. Ashgate Publishing Limited, Aldershot (2006)

12. Cox, A.: Embodying Music: Principles of the Mimetic Hypothesis. Society for Music Theory 17(2), 1-24 (2011)

13. Latulipe, C., Cherry, E., Lottridge, D.: Love, hate, arousal and engagement: Exploring audience responses to performing arts. In: Proceedings of ACM CHI 2011. pp. 1845-1854 (may 2011). https://doi.org/10.1145/1978942.1979210

14. Leiner, D., Fahr, A., Früh, H.: Eda positive change: A simple algorithm for electrodermal activity to measure general audience arousal during media exposure. Communication Methods and Measures 6, 237-250 (dec 2012). https://doi.org/10.1080/19312458.2012.732627

15. Likert, Rensis: A Technique for the Measurement of Attitudes. No. 140 in Archives of Psychology, New York (1932)

16. Martella, C., Gedik, E., Cabrera-Quiros, L., Englebienne, G., Hung, H.: How was it?: Exploiting smartphone sensing to measure implicit audience responses to live performances. In: Proceedings of the 23rd ACM International Conference on Multimedia. pp. 201-210. MM '15, ACM, 
New York, NY, USA (2015). https://doi.org/10.1145/2733373.2806276, http://doi.acm.org/10.1145/2733373.2806276

17. Stevens, C., Glass, R., Schubert, E., Chen, J., Winskel, H.: Methods for measuring audience reactions. In: Proceedings of the inaugural International Conference on Music Communication Science. pp. 5-7 (2007)

18. Stevens, C., Schubert, E., Haszard Morris, R., Frear, M., Chen, J., Healey, S., Schoknecht, C., Hansen, S.: Cognition and the temporal arts: Investigating audience response to dance using pdas that record continuous data during live performance. International Journal of Human-Computer Studies 67, 800-813 (sep 2009). https://doi.org/10.1016/j.ijhcs.2009.06.001

19. Swarbrick, D., Bosnyak, D., Livingstone, S.R., Bansal, J., Marsh-Rollo, S., Woolhouse, M.H., Trainor, L.J.: How live music moves us: Head movement differences in audiences to live versus recorded music. Front. Psychol. 9(2682) (jan 2019). https://doi.org/10.3389/fpsyg.2018.02682

20. Wang, C., Geelhoed, E.N., Stenton, P.P., Cesar, P.: Sensing a live audience. In: Proceedings of the SIGCHI Conference on Human Factors in Computing Systems. pp. 1909-1912. CHI '14, ACM, New York, NY, USA (2014). https://doi.org/10.1145/2556288.2557154, http://doi.acm.org/10.1145/2556288.2557154

21. Wigham, P., Böhm, C.: Bazerbows: Instrument design and mimetic theory. Scottish Music Review 4 (2017), http://www.scottishmusicreview.org/ 